\title{
Aromatics from Lignin through Ultrafast Reactions in Water.
}

\author{
Nerea Abad-Fernández ${ }^{\mathrm{a}}$, Eduardo Pérez ${ }^{\mathrm{b}}$ and María José Cocero ${ }^{\mathrm{a}^{*}}$
}

a Bioeconomy Research Institute BioEcoUVa. Research group High Pressure Processes. EII, Sede Mergelina, 47011 Universidad de Valladolid, Valladolid, Spain.* mjcocero@iq.uva.es

b Bioeconomy Research Institute BioEcoUVa. Research Group TERMOCAL, Thermodynamics and Calibration, EII, Sede Cauce, 47011 Universidad de Valladolid, Valladolid, Spain.

Nowadays, the valorization of lignin, the major natural source of aromatics in earth, is being a challenge for the scientific community. In this study, kraft lignin is effectively converted into aromatic monomers by ultrafast depolymerization in hot and pressurized water. At reaction times below $500 \mathrm{~ms}$, it is possible to avoid char formation originated from undesirable condensation reactions by controlling accurately the reaction time. Under alkaline medium, the reaction reaches an optimum point at $386^{\circ} \mathrm{C}$ and $300 \mathrm{~ms}$ with a light oil yield of $60 \%$ with a concentration in key compounds such as guaiacol, creosol, vanillin and acetovanillone of around $20 \% \mathrm{w} / \mathrm{w}$. The total aromatic monomeric yield based on lignin was $10.5 \% \mathrm{w} / \mathrm{w}$. The char formation in this point was surprisingly low (4 $\% \mathrm{w} / \mathrm{w})$. Analysis and quantification of the products allows to identify the evolution of the different reaction steps and propose plausible mechanism for the depolymerization and repolymerization stages. Furthermore, it is proven that the proposed technology is equally effective to treat directly industrial black liquors with a yield higher than $50 \%$ to light oil, containing as main monomers guaiacol $(2.7 \%)$, syringol $(3.0 \%)$ and syringaldehyde $(7.3 \%)$.
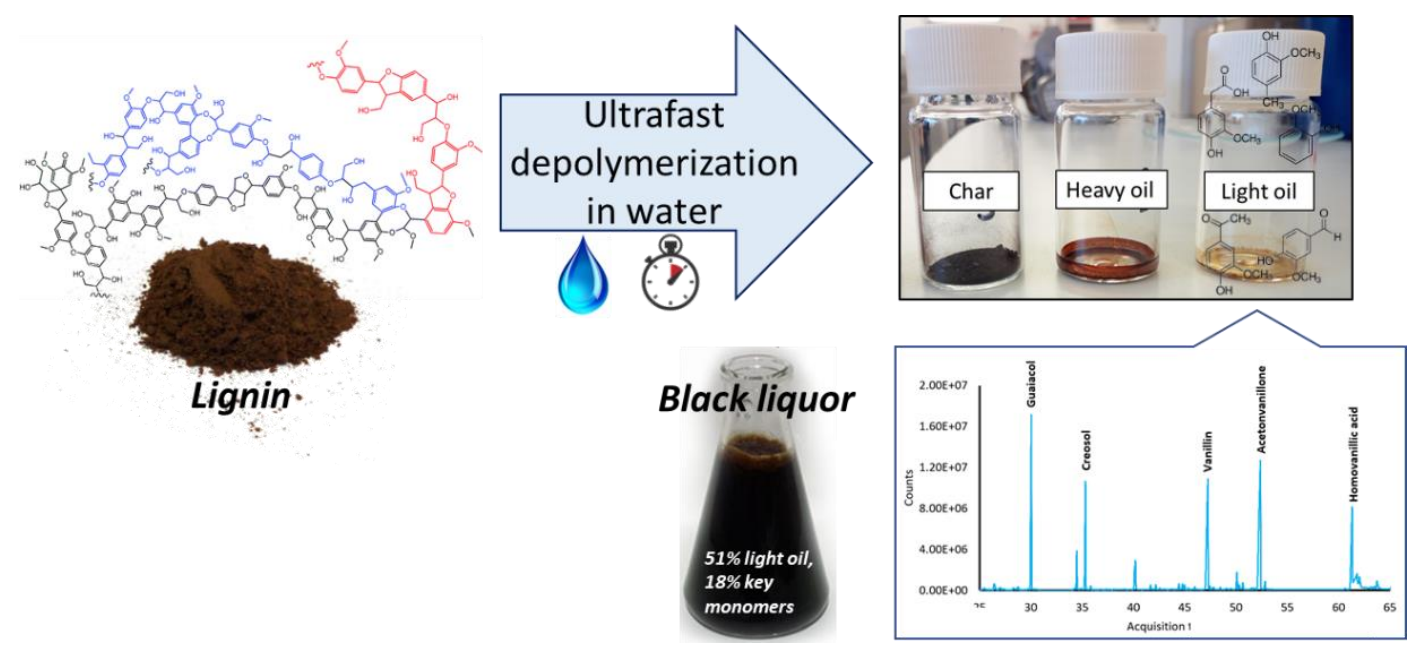

Key words: High-pressure chemistry, Lignin, Microreactors, Radical reactions, Reaction mechanisms. 


\section{Introduction}

Bio-based polymers' growth rates are at same level as global polymers: Worldwide production capacity is forecasted to increase from 6.6 million tons in 2016 to 8.5 million in 2021. In the absence of renewables aromatic building blocks, polymers are currently produced from a petrochemical aromatic molecule and a bio-based aliphatic molecule. ${ }^{1}$

Lignin is an aromatic biopolymer that constitutes a major component of lignocellulosic biomass. It is composed by combination of three phenylpropanoid units (p-coumaryl, coniferyl and synapyl alcohols) randomly connected in different proportions depending on the biomass of origin. Despite its abundance and promising molecular structure for obtaining high value monoaromatic compounds, lignin is still underutilized compared to other feedstocks ${ }^{2}$. Currently, lignin is produced in large quantities in the pulp and paper industry as the main component of black liquor, a low-value residual stream that is further processed to be burned and obtain energy in the same plant. Moreover, with the increasing role of biorefineries in a bio-based economy, even more generation of lignin is expected.

Depolymerization is an obvious strategy to valorize lignin as it provides single aromatic chemicals. However, this route has been deceptive because of the low selectivity of the compounds targeted and the tendency of lignin fragments to recombine to form char-type by-products. ${ }^{2}$ However, this has not discouraged the scientific community who has redoubled its efforts to obtain a solution and as consequence, important advances have been achieved. Some of the most successful approaches explored were catalyst improvement $^{3}$,or pretreatment stages with oxidants ${ }^{4}$ or formaldehyde ${ }^{5,6}$. Nevertheless, this kind of meticulous process are very far of being translated into commercial practice due to the several non-continuous steps that must be carried out and the long time required. Ragauskas et al. ${ }^{7}$ have presented this paradigm, posing the reality: "although fundamental research has historically focused on converting lignin to chemicals materials, very little of this effort has been translated into commercial practice". So, what has changed to address this deadlock?

An answer to it could be the continuous hydrothermal depolymerization approaches ${ }^{8}$ possess certain advantages such as the cost and safety of the solvent or, since 
the black liquor containing lignin is already in aqueous phase, it can be directly treated. The depolymerization products can then be fractionated to be utilized as a mixture while added-value components are isolated. ${ }^{2,9}$ The most common versions of hydrothermal lignin depolymerization include hydrothermal pyrolysis/hydrolysis, ${ }^{10,11}$ base catalyzed depolymerization (BCD), ${ }^{12,13}$ hydrogenolysis ${ }^{14}$ and oxidative cleavage ${ }^{2,9}$ in sub- and supercritical water. These techniques require high temperatures and therefore they imply an additional energetic cost but on the other hand, reaction kinetics is enhanced reducing the reaction times so that it allows a feasible scale-up of the process with small reactor volumes, i.e. intensification of the process. Some studies have demonstrated that residence times of the order of seconds are enough to convert most of the lignin to a mixture of products. ${ }^{2}$

However, as temperature is raised, a phenolic char is preferentially formed through crosslinking of phenolic units and formation of recalcitrant $\mathrm{C}-\mathrm{C}$ aromatic to aromatic bonds. ${ }^{15}$ These undesirable condensation reactions seem to take place rapidly, at rates comparable to those the depolymerization reaction so that it is not possible to control them by conventional batch reactors. Yong and Matsmura ${ }^{11}$ have achieved high lignin conversion of alkali lignin at reaction times as low as 0.5 second in a continuous apparatus in SCW conditions, but still obtaining a high amount of char due to repolymerization reactions. Thus, it is necessary to work at reaction times short enough to determine the reaction time in which the repolymerization of lignin starts, avoiding the problem present until today and obtaining high yields and selectivities towards monoaromatics, with a low char formation.

Achievement of accurately time-controlled reactions in supercritical water at the range of milliseconds can be achieved using the Sudden Expansion Micro-Reactor (SEMR), developed in our laboratory. ${ }^{16}$ The keys of this design are very small continuous reactors and an instantaneous cooling step achieved by sudden decompression thanks to the Joule-Thompson effect. This way, residence times down to $40 \mathrm{~ms}$ can be reached with an uncertainty of $\pm 1 \mathrm{~ms}$. Despite being a bench-scale plant, it can reach a treatment capacity of $3 \mathrm{~kg} / \mathrm{h}$ of solution. To increase the concentration of lignin, $\mathrm{NaOH}$ must be added to favour the solubility of the reaction products and to avoid blockages, with the additional advantage that it acts as catalyst. ${ }^{2}$ 
To facilitate understanding and analysis of the reaction products, it has been common to fractionate the samples according to their solubility in water and organic solvents ${ }^{2,10,13}$. As polar solvents would dissolve similar solutes, lignin fragments from lignin breakdown would distribute within the solvents according to their chemical characteristics. Ethyl acetate seems to be a good candidate as extracting solvent as it is non-costly and environmentally benign. Thus, a light fraction can be extracted from the water-soluble compounds, namely light oil, mainly composed by aromatic fragments from depolymerization. The water-insoluble compounds can likewise be fractionated into a heavy oil and a solid residue that has been commonly designated as char.

2. Results and discussion

2.1. Effect of temperature and reaction time on the kraft lignin depolymerization.

The light oil fraction mainly consists of a mixture of oligomers, ${ }^{2}$ but it also concentrates the monomeric products, with a good selectivity, as detected by GC-MS (See Table 1 and Figure 7). Thus, it can be envisaged as a desired crude depolymerization product since monomers can be more easily isolated from the remaining mixture of oligomers and the latter be used as precursor of materials. ${ }^{9}$ Optimization of this oil fraction depends on time and temperature (Figure 1) as maxima are found at longer reaction times for lower temperatures. However, it seems that reaction time has a more detrimental effect than temperature as the highest yield of light oil was obtained at 400 ${ }^{\circ} \mathrm{C}$ and short times. These results are in agreement with other studies where it is shown that increasing the reaction time (i.e. increasing the reaction severity), decreases the concentration of phenolic compounds (oil production) as the importance of recondensation reactions increases. ${ }^{15}$ 


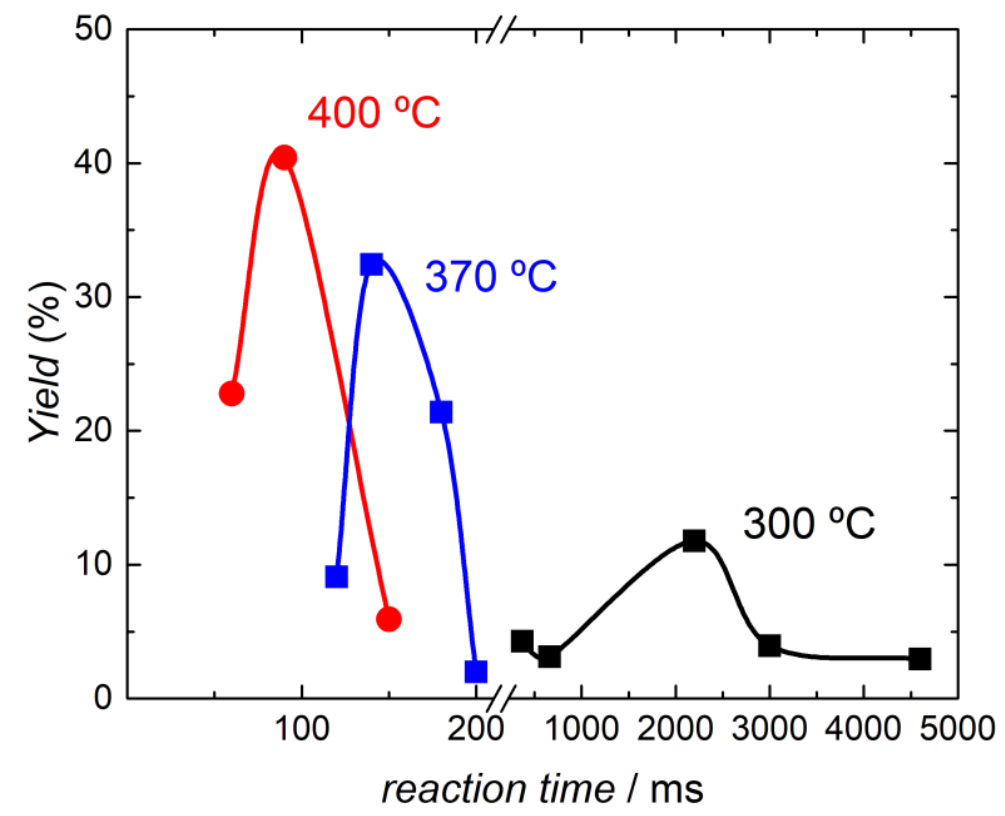

Figure 1. Depolymerization of lignin in sub and supercritical water. Light oil yield vs reaction time at different temperatures.

\subsection{The catalytic effect of $\mathrm{NaOH}$}

Hydrolysis of lignin can be enhanced using bases as catalyst. ${ }^{2,12,13}$ In figure 2 some proposed mechanisms of the catalytic role of bases are represented. According to literature, a base can help cleavage of the $\beta-\mathrm{O}-4$ ether bond via nucleophilic attack of an adjacent methoxy group, ${ }^{17}$ or heterolytically via a six-membered transition state ${ }^{13}$ (reactions $\mathrm{a}, \mathrm{b}$ in figure 2 respectively): A base can also promote propagation reactions during a radicalary mechanism ${ }^{18}$ (reaction c). However, most of these studies have been done at water well below the critical point; one of the reasons may be that due to the ionic nature of the catalysts, conditions of high ionic product are required. 


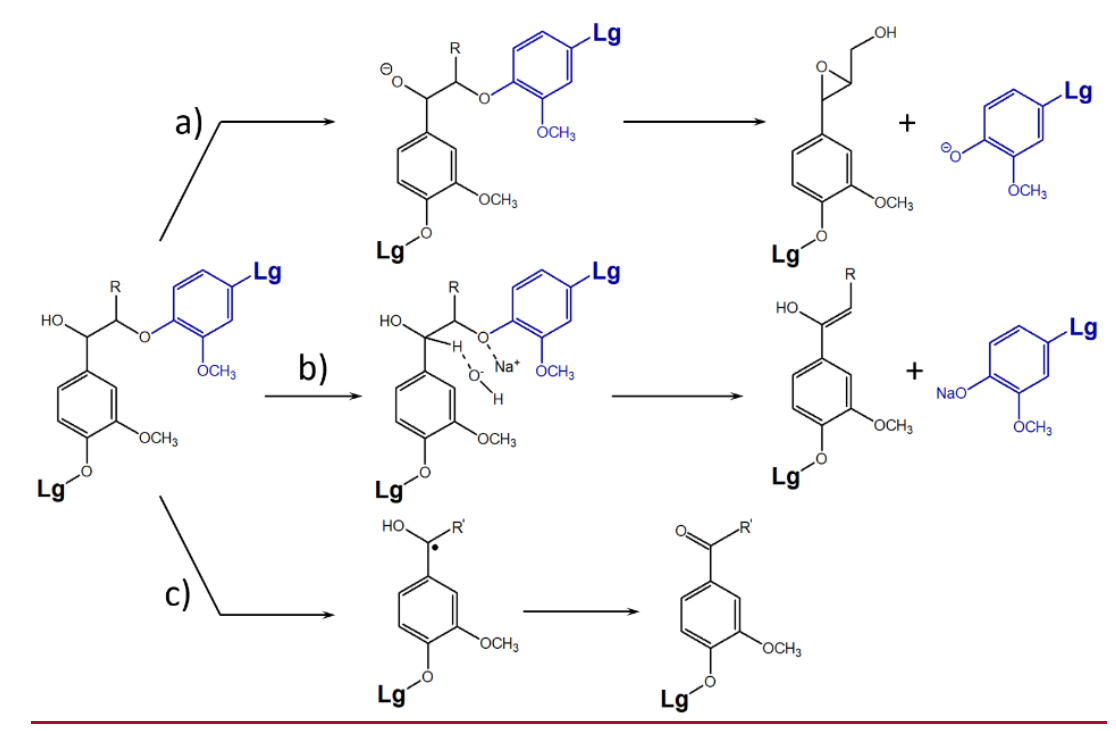

Figure 2. Possible mechanisms of $\mathrm{NaOH}$ acting as catalyst for the depolymerization of lignin.

However, a catalytic effect for $\mathrm{NaOH}$ has also been observed at supercritical temperatures, where ionic pathways are supposed to be less important, and at reaction times of several seconds. ${ }^{2}$ The same has been observed in this work at the range of milliseconds. Experiments done at $386^{\circ} \mathrm{C}$ in absence of catalyst yielded a maximum of $45 \%$ in aromatic oil (see Figure S3) while the maximum yield was $67 \%$ with $\mathrm{NaOH}$. Similarly, the maximum yield in monomers was only $4.5 \%$ without $\mathrm{NaOH}$ in contrast to $10.5 \%$ using catalyst. Thus, at these conditions some of the mechanism proposed must also have a significant role during depolymerization.

It is well known that due to the low dielectric constant of supercritical water, reaction mechanisms that imply free ionic species such mechanism a in figure 2 are disfavored. For the same reason, it is accepted that lignin depolymerization mechanisms proceed via radicalary pathways in supercritical water. Reaction b, even being a heterolyitic pathway, it does not imply free ionic species so it may occur in SCW. Reaction c itself does not describe a direct influence of $\mathrm{NaOH}$ in cleavage of ether bonds but a base would help the propagation of the radicalary reaction and the stabilization of the monomers and thus, it would diminish repolymerization. Indeed some authors suggest that even at supercritical conditions, a combination of both ionic and radicalary mechanisms is possible. ${ }^{19}$ 


\section{3.- Kraft lignin depolymerization vs reaction time. Mechanisms}

Figure $3 \mathrm{a}$ show the yield of aromatic oil and monomers obtained at different reaction times. An optimum point can be detected for the depolymerization of lignin at $386^{\circ} \mathrm{C}$ using $\mathrm{NaOH}$ as catalyst. At reaction times of $240 \mathrm{~ms}$ the yield of light oil is maximum and decreases progressively as reaction proceeds. Monomers are produced at longer reaction times as the maxima is obtained at $300 \mathrm{~ms}$. The main monomers obtained are guaiacol, vanillin, acetovanillone and homovanillic acid and they seem to be produced simultaneously (see figure S5) The shape of the graphic clearly suggests that monomers are produced from oligomers, which are the main constituents of the light oil. For reaction times longer than $300 \mathrm{~ms}$, a dramatic decreasing of both monomer and light oil yields is observed accompanied by an increment of solid fractions (figure $3 \mathrm{~b}$ ), which clearly points to a degradation of the products by recombination reactions. ${ }^{2}$

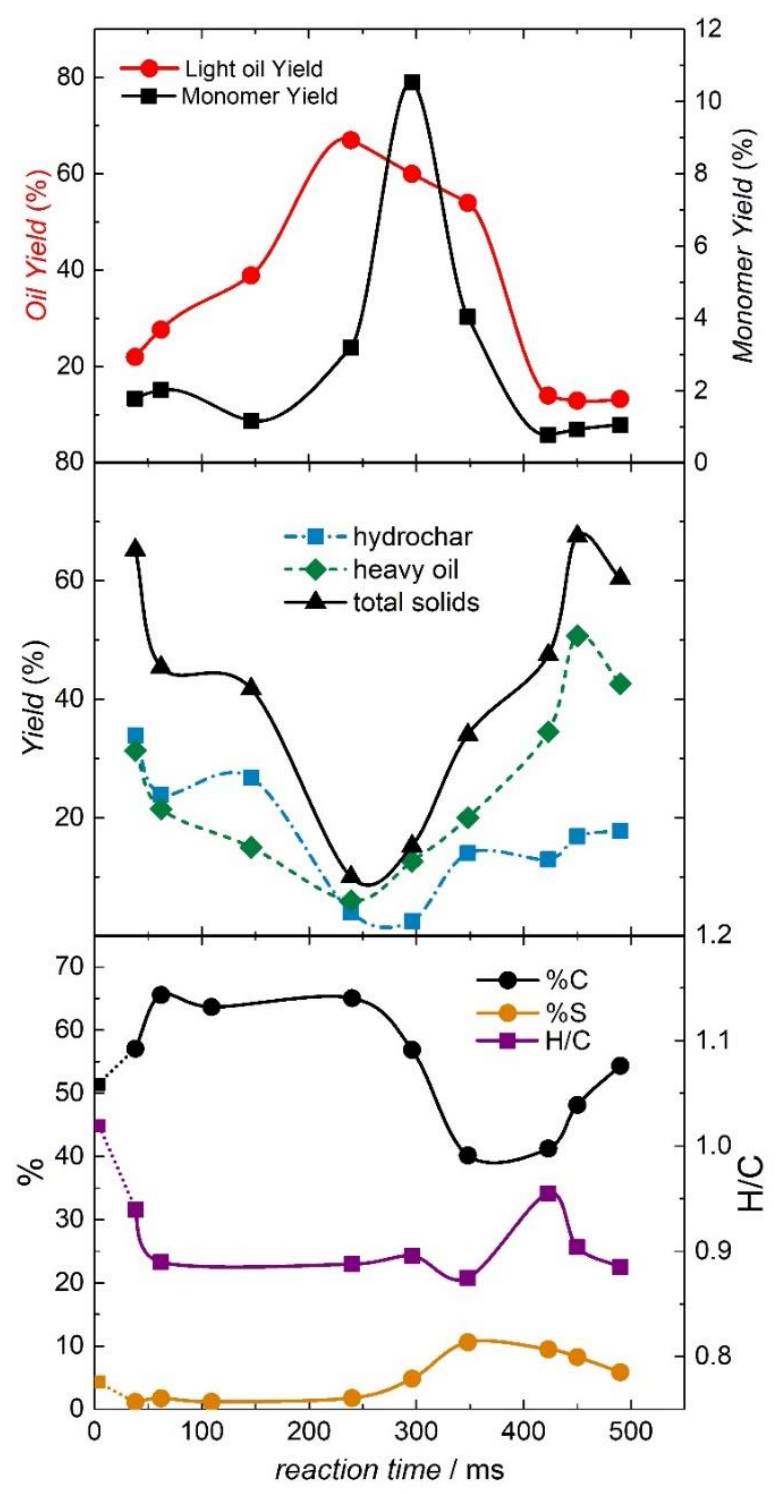


Figure 3. Evolution of the depolymerization reaction of kraft lignin vs time at $386{ }^{\circ} \mathrm{C}$ with $\mathrm{NaOH}$ catalyst addition. a) Light oil and monomer yield. b) Yield of the solid fractions (heavy oil and char) with $\mathrm{NaOH}$ as catalyst. c) Elemental composition for the char.

However, an anomalously high yield of the solid fractions at times below $240 \mathrm{~ms}$ was really surprising, reaching a minimum at the point of maximum light oil yield. This behavior was also observed at other temperatures (figure S4). As condensation products do not seem to undergo further depolymerization, ${ }^{11,13,20}$ water insoluble fractions obtained at reaction times below or over 240 ms must be different in composition than each other and therefore, originated by different kind of reactions. Indeed, TGA done to these samples reveal significant differences within them and the starting lignin.

Figure 4a show the derivative TGA for kraft lignin and two hydrochars at reaction times lower and higher than the optimum point. Peaks below $150^{\circ} \mathrm{C}$ are usually associated to water adsorbed to the structure so the latter contain much less moisture that the former, which may indicate that hydrochars are less hygroscopic. Lignin decomposition profile present a major peak at $320^{\circ} \mathrm{C}$, associated to the loss of $\mathrm{H}_{2} \mathrm{O}$ and formaldehyde from aliphatic hydroxide moieties. ${ }^{21}$ These peaks appear in hydrochars at higher temperatures $\left(400-420^{\circ} \mathrm{C}\right)$ and are smaller, probably coming from decomposition of other oxygencontaining groups, since the aliphatic $-\mathrm{OH}$ seem to have been lost.

There is a main difference, however between the two hydrochars, the one obtained at longer reaction seem to present much more stable moieties than that obtained at the beginning of the reaction as the former has a peak in the derivative at $T=770{ }^{\circ} \mathrm{C}$ which is coherent with the presence of resilient condensation products. However, this hydrochar also present some groups that decompose at low temperature $\left(T \sim 250^{\circ} \mathrm{C}\right)$, which could be attributed to a loss of $\mathrm{CO}_{2}$ via decarboxylation. ${ }^{21}$ 

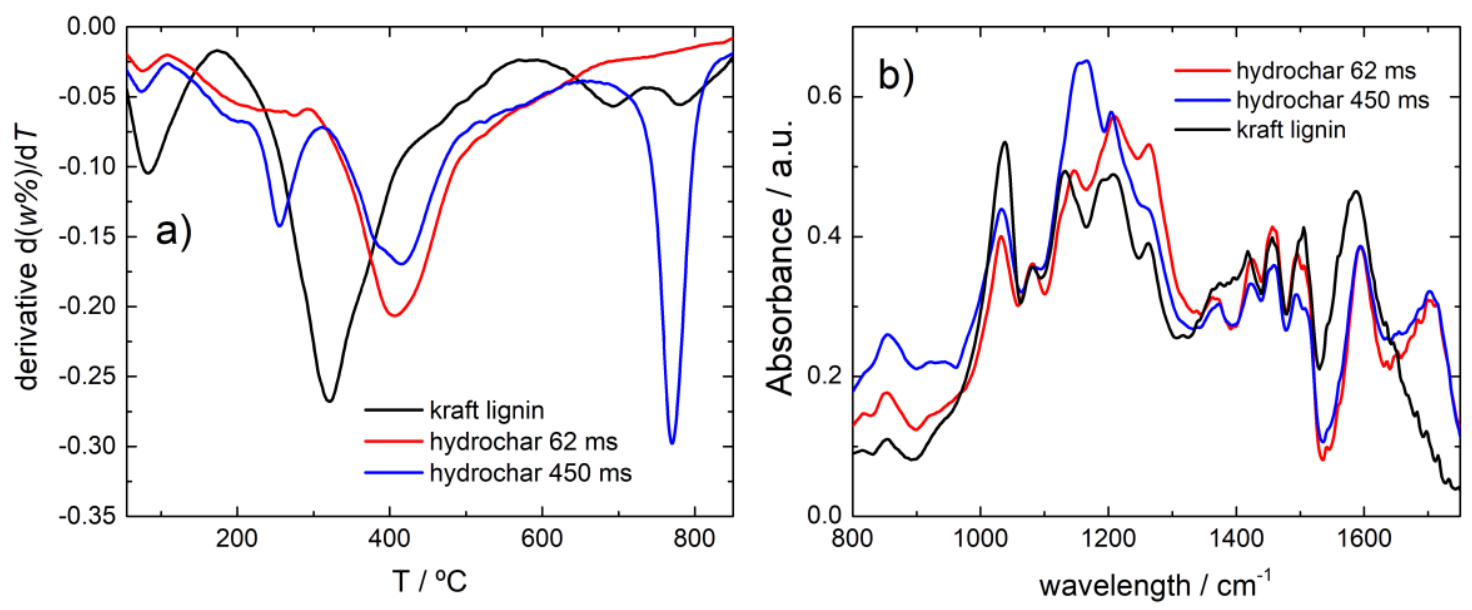

Figure 4. Comparison between chars at $62 \mathrm{~ms}$ and at $450 \mathrm{~ms}$ reaction time and the starting lignin. Temperature was $386^{\circ} \mathrm{C}$. a) Thermo Gravimetrical Analysis (derivative). b) FTIR spectra.

Other analysis such FTIR reveal certain chemical characteristics of these fractions. Whatever transformations are occurring they do not alter significantly the aromatic structure as little difference is observed in the FT-IR spectrum at wavenumbers within 1400 and $1650 \mathrm{~cm}^{-1}$ (figure 4b). This is not the case of the C-O bonds whose signals appear within 1000 and $1300 \mathrm{~cm}^{-1}$ as spectra of lignin, and solid at short and long residence times are different. Another significant difference between hydrochar and kraft is the presence of carbonyl groups in the former (range $1650-1750 \mathrm{~cm}^{-1}$ ). They are most likely originated from the aliphatic $-\mathrm{OH}$. Previous reports have shown how these latter moieties are practically absent in hydrochars upon hydrothermal treatment of lignin. ${ }^{2}$ These fractions also seem to contain in their structure a higher proportion of $\mathrm{C}=\mathrm{C}$ double bonds $\left(800-900 \mathrm{~cm}^{-1}\right)$ than in the starting lignin or the rest of the fractions (figure $\mathrm{S} 10$ ).

Elemental analysis provides certain chemical information about the sample as a whole ${ }^{2}$, particularly the ratio $\mathrm{H} / \mathrm{C}$ as it is an indicator of the degree of unsaturation (proportion of double bonds, aromatic rings and cycles). In these reactions, elemental analysis shows a significant change in $\% \mathrm{C}, \% \mathrm{H}$ and $\% \mathrm{O}$ respect the starting kraft lignin (figure 3c). The values of $\mathrm{H} / \mathrm{C}$ quickly decrease within the first $64 \mathrm{~ms}$ while those for $\% \mathrm{C}$ increase. We propose then that at the very first stages of the reaction different reactions that do not lead to lignin depolimerization occur very rapidly. These reactions would convert $-\mathrm{OH}$ to moieties with a higher degree of unsaturation. For example, dehydration reactions $^{22}$ (Figure 5, reaction $\mathbf{1} \leftrightarrow 4$ ) that would explain the increment of \%C and decreasing of $\mathrm{H} / \mathrm{C}$ in elemental analysis. Another possibility could be base-catalyzed 
formation of carbonyl groups from hydroxyl (reaction c in figure 2 and analogous) that would yield $\alpha$ - carbonyl structures as observed in FTIR. Dehydration reactions have been observed in water at both sub- and supercritical conditions. It is well known that below the critical point, dehydration proceeds via ionic pathways, ${ }^{23}$ but at higher temperatures a radicalary (pyrolytic) mechanism is possible. ${ }^{22}$

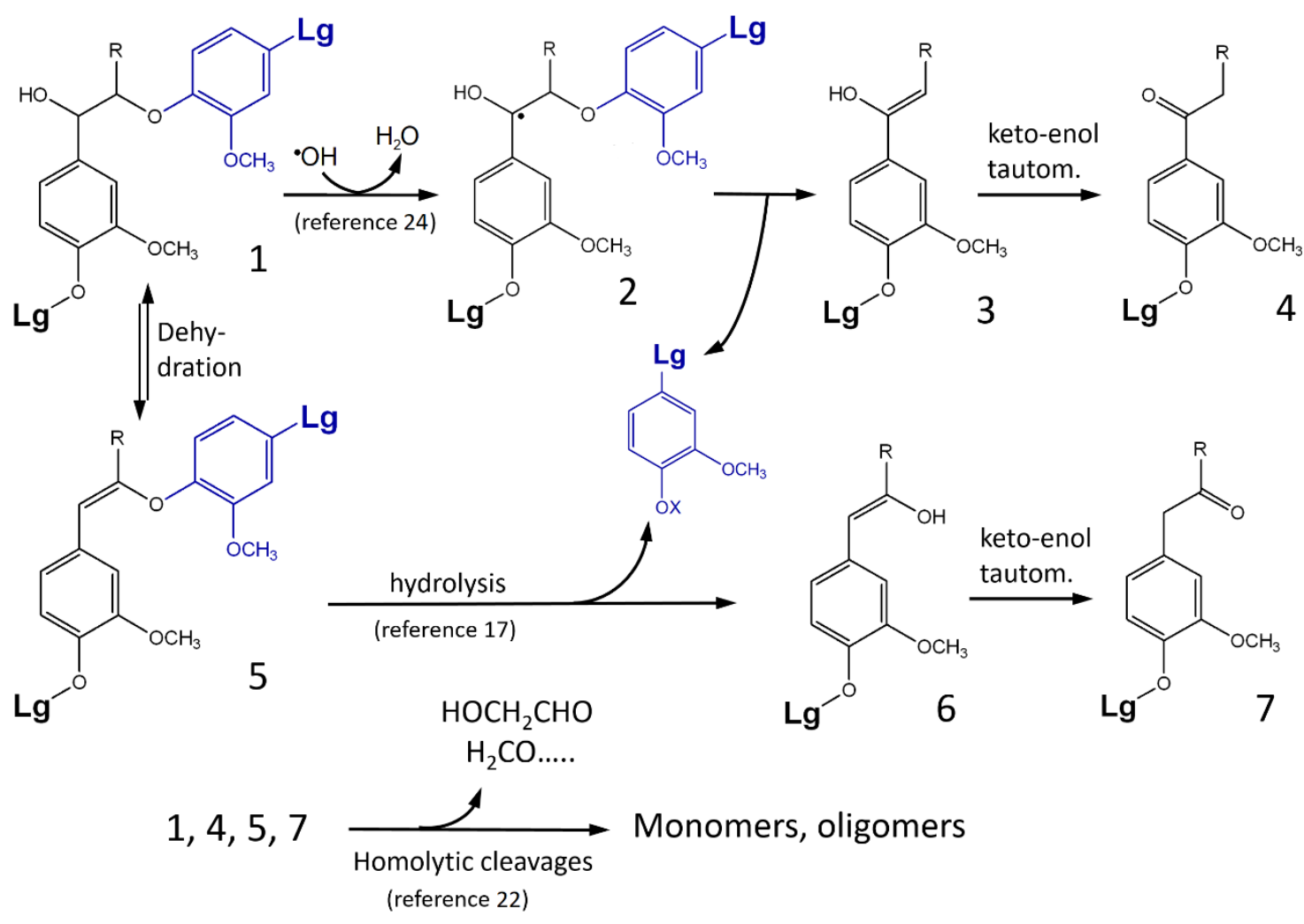

Figure 5. Proposed reaction scheme for the aromatic products from kraft lignin in SCW.

This hypothesis can be further supported by Heteronuclear Single-Quantum Correlation (HSQC), which is a 2D NMR technique that detects ${ }^{13} \mathrm{C}-{ }^{1} \mathrm{H}$ coupling separated by three or four bonds. The - $\mathrm{OH}$ groups located in the aliphatic backbone of lignin disappear upon SCW treatment (Figure S12). A loss of -OH groups, implies a decreasing solubility for lignin in water, thus being accumulated in the solid fractions, which would explain why such apparent high yield of char at short reaction times.

Reactions leading to depolymerization are slower and they are observed at the time ranges studied in this work. (64-300 ms). As explained above, hemolytic cleavages are more probable to occur in supercritical water but heterolytically pathways should not be driven out. A summary of possible mechanisms is displayed in figure 5. A radicalary mechanism for $\beta-\mathrm{O}-4$ cleavage under pyrolytic conditions has been proposed: ${ }^{24}$ (2) is 
formed by H-abstraction. This reaction followed by a keto-enol tautomerization $(\mathbf{3} \rightarrow \mathbf{4})$ would also lead to $\alpha$-carbonyls, first oligomeric and then monomeric compounds (vanillin and acetovanillone). Notice that enolate (3) is also produced heterolitically base-catalized (reaction b, figure 2) which may occur paralelly. It was also proposed in reference 24 that the typical initiation reactions favour an initial dehydration of lignin structures that results in an increment of the unsaturation degree, as observed in this work.

Subsequently, more $\beta$-O-4 ether linkages will be progressively cleaved and smaller oligomers and monomers will be incorporated to the aromatic oil fraction increasing its yield while the amount of solid fraction decreases down to an optimum point at $240 \mathrm{~ms}$ for oligomers and a bit longer $(300 \mathrm{~ms})$ for monomers. The fact that all the major monomers are obtained simultaneously suggest that they are directly produced from the oligomers.

However, it is clear that apart from breaking ether linkages, depolymerization mechanisms must break C-C bonds since, the mayor monomers obtained in this work and throughout the literature are formed by an aromatic unit and C2, C3 or no sidechain., ${ }^{2-}$ 11, 13, 20 Thus, the mechanisms to produce the main monomers must include homolytic cleavages. Vanillin and acetovanillone are two of the main products obtained from lignin depolymerization in high-temperature water. They may arise from $\mathbf{2}$ or $\mathbf{4}$ by radicalary reactions similar to those observed in glycerol reactions in $\mathrm{SCW}{ }^{22}$ (see figure S13). Guaiacol (or its counterpart syringol) is also a major depolymerization product. It has been proposed to be formed from vanillic acid decarboxylation ${ }^{2,25}$ but at the short reaction times covered in this work, that is unlikely as this reaction follows a slower kinetics. The presence of guaiacol can then be explained by a radicalary mechanism of Ar-C bond cleavage that is known to happen in lignin pyrolysis. ${ }^{24}$ The presence of homovanillic acid is somewhat more difficult to explain as the kind of monomers containing a methylene group in $\alpha$ have never been detected. The dehydrated structure of lignin (5) can be followed by a depolymerization step by hydrolysis, ${ }^{17}$ generating an enolate (6) that will immediately proceed to form the corresponding ketone via keto-enol tautomerization. The resulting $\beta$-carbonylic moiety (7) could be further oxidized to homovanillic acid. Hints of another compound never identified before (3-guaiacyl propenal), which could be a precursor of other monomers, have been detected at shorter times (109 ms). But more evidences are necessary to confirm this (see supplementary information for further details). 


\section{4.- Repolymerization reactions}

As discussed above, it is likely that hydrochar obtained at lower reaction times is originated from dehydration reactions which would not supose degradation of the polymeric material as these solid fractions would be sequentially converted to aromatic oil and monomers. However, at reaction times higher than $240 \mathrm{~ms}$, the tendency changes and the amount of solids increases again following mechanisms of repolymerization of lignin monomers and oligomers to higher molecular weight compounds, as proposed in literature. ${ }^{10,13}$ This repolymerization was confirmed using Gel Permeation Chromatography. Figure 6a shows GPC traces for the fractions obtained at $348 \mathrm{~ms}$. Hydrochar appears at shorter retention time (RT) than kraft lignin, i.e. has a higher average molecular weight, while heavy and light oils are smaller molecules. Figure $6 \mathrm{~b}$ shows the maxima of GPC chromatogram for the hydrochar at different reaction times. Below $240 \mathrm{~ms}$, the average size of the hydrochar molecules is similar to that of the kraft lignin but at longer times, the hydrochar molecules are significantly bigger indicating that it has been originated by repolymerization.
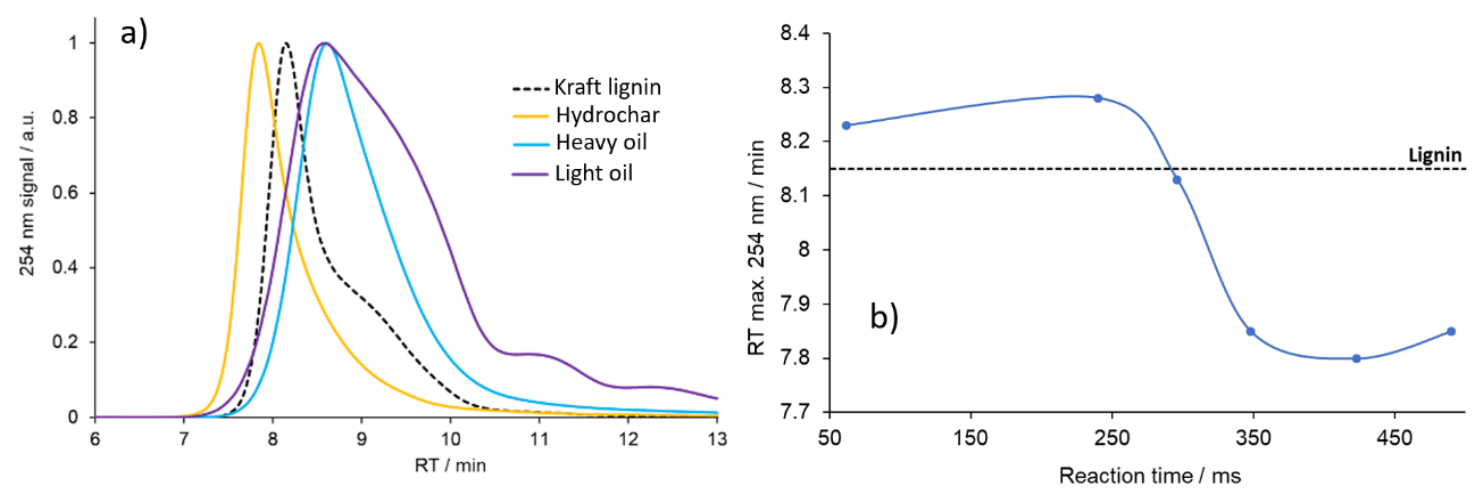

Figure 6. a) GPC curves for the fractions obtained after depolymerization of kraft lignin at $348 \mathrm{~ms}$. b) Curve of retention times at which signal is maximum for the hydrochars at different reaction times. The dotted line represents the starting kraft lignin.

The mechanisms of lignin repolymerization are still not clear although they are very likely to dependent on the reaction conditions. Roberts et al. ${ }^{19}$ propose that during base catalyzed depolymerization at subcritical water condensation reactions proceed via carbanion addition. Shuai and Saha, ${ }^{15}$ however support the idea that small fragments released during depolymerization ( $\mathrm{C} 1$ or $\mathrm{C} 2$ aldehydes) are the responsible of these reactions. 
We have conducted some experiments reacting equimolar amounts of vanillin and acetovanillone as model monomeric compounds under the same conditions than in lignin depolymerization $\left(386{ }^{\circ} \mathrm{C}\right.$, reaction times between 250 and $700 \mathrm{~ms}$ ). Surprisingly both vanillin and acetovanillone do not degrade significantly upon SCW treatment, concluding that the degradation of monomers during the reaction is caused by other species inherent to the reaction mechanism such as the small aldehydes abovementioned or other radicalary intermediates. These observations also confirm that the carbanion-addition mechanism does not apply at the conditions explored in this work. In any case, due to the lability of monomers within this reactive environment, controlling the reaction time is essential to avoid degradation of the latter.

However, not much information about the chemical composition of hydrochars has been reported, so the kinetics and mechanisms of the reaction remain speculative. Elemental analysis of hydrochar (over $240 \mathrm{~ms}$ ) provided additional clues about the evolution of the reaction as two distinct stages can be clearly distinguished: initially (between 300 and $420 \mathrm{~ms}$ ), progressive decreasing of \%C accompanied by an increment in $\mathrm{H} / \mathrm{C}$ ratio to values more typical of the aromatic oil is observed, that is indicative of a repolymerization by coupling from the components of monomers and oligomers. ${ }^{13,15,17}$ At the later steps of the reaction $(>400 \mathrm{~ms})$, this tendency is again reversed probably due to further reactions of carbonization, loss of $-\mathrm{OH},{ }^{10}$ or demetylation ${ }^{2}$ and that may be responsible of the behavior of the TGA traces at high temperature.

\section{5.- The fate of sulfur}

An interesting observation is the figure for $\% \mathrm{~S}$. Its concentration in char is kept low and steady until the repolymerization process starts. Then sulfur, presumably from the starting lignin, is progressively incorporated to this fraction up to values $10.6 \%$ (approximately $35 \%$ of the total sulfur originally contained in kraft lignin). One can hypothesize the formation of analogous thioaldehydes and thioketones from - $\mathrm{SH}$ substituted units of kraft lignin that will readily incorporate to the condensed structure as they are not stable and are known to aggregate. More detailed studies are necessary to determine the fate of sulfur in technical lignins, as the mechanisms involved seem to be strongly dependent on the type of the sulfur moiety. For example, it has been observed that, unlike sulfide moieties, sulfonate groups present in lignosulphonates, do not incorporate to char or other fractions but they tend to remain in the aqueous residue as partially reacted lignin or free sulfate. ${ }^{2}$ Determining the behavior of sulfur-containing 
moieties in technical lignins is of crucial importance from the practical point of view, as they are often undesired when using lignin or lignin derived compounds as precursors for the synthesis of other materials such as active carbon or polyurethane foams. ${ }^{9}$

\subsection{Supercritical water depolymerization of a black liquor with low content of lignin.}

Nowadays, lignin residual streams like black or brown liquors, which are obtained as residues in some industries (i.e. pulp and paper industries), impose significant technical challenges to be used in high value applications. Kraft black liquors are further processed in the same pulping plant to recover the inorganic reactants. Very often, lignin production exceeds the necessities of the pulping plant, so the remaining material is burned to generate electric energy. Thus, black liquor can be considered a low-value by-product and direct valorization of it would be very desirable. In this work we demonstrate that ultrafast depolymerization in supercritical water is also applicable to these industrial streams, which would be a more advantageous process because the steps of lignin isolation are no longer necessary. Moreover, the black liquor stream from Kraft process itself has a basic $\mathrm{pH}$ of 12-13, which may act as catalyst.

Isolation of lignin from black liquors is industrially achieved by precipitation by acidification using sulfuric acid or $\mathrm{CO}_{2}$. In the process proposed in this work a depolymerization step is included before acidification so it can yield an added-value additional fraction rich in monoaromatics. A continuous process with microreactors can be very easily scaled-up. ${ }^{26}$ Moreover, the costs associated to the SCW treatment could be minimized by proper energetic integration. ${ }^{27}$ Then, a carefully optimized procedure for purification of the components would be the key for the economic feasibility of the overall process. For this, there are opportunities to improve as many approaches to purify the mixture have been proposed, ${ }^{28}$ for example, supercritical $\mathrm{CO}_{2}$ can be used to both acidify and extract the monomers from the reacting mixture ${ }^{29}$ or from the light oil. ${ }^{9}$

A black liquor supplied by Técnicas Reunidas S. A. (Spain) containing $17.2 \%$ of lignin was depolymerized for $300 \mathrm{~ms}$ and with $\mathrm{NaOH}$ as well as the corresponding Kraft Lignin isolated from it (namely Kraft-BL) for comparison. A reaction time of $300 \mathrm{~ms}$ was chosen for performing these experiences since it was the optimal reaction time in terms of both light oil yield and selectivity in the oil in the experiments performed for Kraft lignin depolymerization. The results obtained are shown in Table 1 and they indicate that the supercritical water depolymerization $\mathrm{NaOH}$ catalyzed is feasible, even if lignin is 
contained in a complex mixture as it is black liquors. The light oil yield obtained for the black liquor $(51.3 \%)$ is similar to that obtained from the commercial kraft lignin and significantly higher than the corresponding kraft-BL lignin.

The composition in monomers of this oil expressed in concentration in $\%(\mathrm{w} / \mathrm{w})$ is also seen in table 1. Figure 7 shows the GC-MS traces for the lignins and the black liquor studied in this work, indicating that the monomeric compounds are obtained with a very good selectivity, which is unusual in hydrothermal reactions at such high temperatures. It seems that the short reaction times are not only useful to control the char production but also the selectivity of the monomers.

The main differences observed between chromatograms are related to the starting lignin. Only units derived from coniferyl alcohol were detected from commercial lignin from Sigma, concluding that this must have been originated from softwood. Black liquor and lignin from Técnicas Reunidas, on the contrary, present significant proportions of sinapyl alcohol derives units revealing its hardwood origin. However, depolymerization of Kraft-BL yielded much less amounts of coniferyl alcohol-derived units that the corresponding black liquor. This difference can be due to the modified structure of the Kraft-BL lignin after being extracted from the black liquor. In any case, the direct treatment of the black liquor results again beneficial compared to that of the isolated lignin as coniferyl alcohol derived units are commercially more valuable.

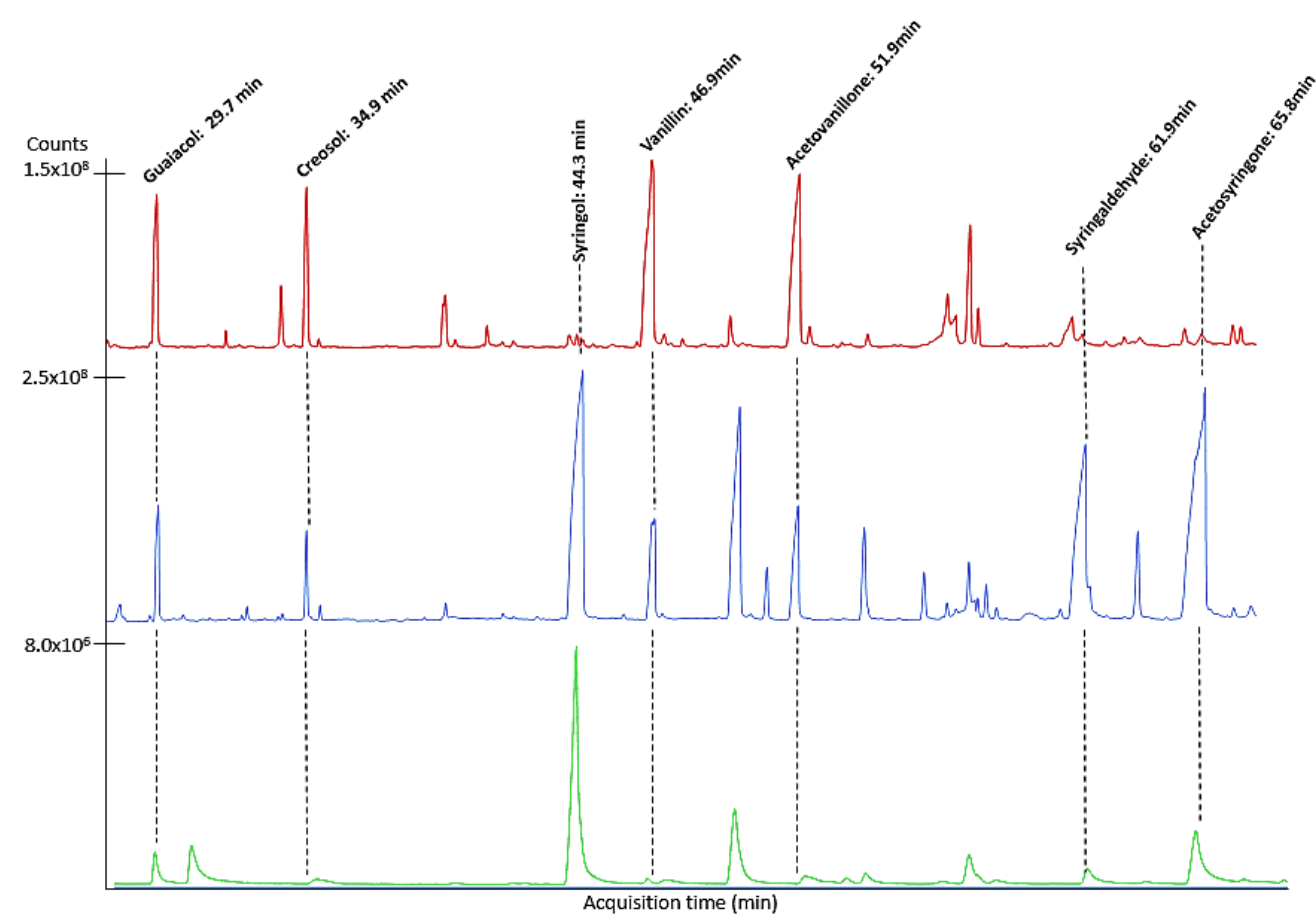


Figure 7. GC-MS chromatograms: light oils obtained at a reaction time of $300 \mathrm{~ms}$ and a temperature of $386^{\circ} \mathrm{C}$ in alkaline medium from: Kraft lignin - Sigma Aldrich (red); Black liquor (blue); Kraft-BL lignin (green).

Table 1.Ultrafast depolymerization of lignin and black liquor using supercritical water (300 ms, $386^{\circ} \mathrm{C}, 260 \mathrm{bar}$ ) in alkaline medium. Light oil yield and composition of the major monomers. The heavy oil contained negligible concentrations of monomers. All values of the aromatic monomers $(\%, \mathrm{w} / \mathrm{w})$ are referred to the aromatic oil production. Values for light oil yields are expressed in base of the lignin concentration at the inlet of the reactor.

\begin{tabular}{|l|c|c|c|}
\hline & Kraft-Sigma & Black Liquor & Kraft-BL \\
\hline Light oil yield & 60.0 & 51.3 & 23.5 \\
\hline Guaiacol & 3.9 & 2.7 & 0.8 \\
\hline Vanillin & 4.1 & 0.6 & 0.3 \\
\hline Acetovanillone & 3.3 & 1.6 & 0.3 \\
\hline Homovanillic acid & 4.2 & 0.0 & 0.0 \\
\hline Creosol & 2.0 & 0.8 & 0,0 \\
\hline Syringol & 0.1 & 3.0 & 4.1 \\
\hline Syringaldehyde & 0.7 & 7.3 & 4.0 \\
\hline Acetosyringone & 1.5 & 1.9 & 3.8 \\
\hline TOTAL & 19.8 & 17.9 & 13.3 \\
\hline
\end{tabular}

\section{Experimental section}

\subsection{Materials}

Lignin used was kraft lignin with low sulfide content (4\% sulfur) from Sigma-Aldrich. Distilled water was used as the reaction medium to run the experiments. Sodium hydroxide used as catalyst was purchased from Sigma-Aldrich. The calibration standards used in the GC-MS analysis were all purchased from Sigma-Aldrich (USA): syringol ( $\geq 98.0 \%$ ), acetosyringone (97\%), homovanillic acid ( $\geq 97.0 \%)$, guaiacol $(\geq 99 \%)$, creosol ( $\geq 98 \%$ ), acetovanillone ( $\geq 98.0 \%$ ), vanillin (99\%), syringaldehyde ( $\geq 97.0 \%)$ and the internal standard 2-phenyl ethanol $(\geq 99.0 \%)$. Ethyl acetate $(99.8 \%)$ employed for carrying out the extractions was purchased from Sigma Aldrich. Technical kraft lignin 
named as Kraft-BL and the corresponding black liquor were supplied by Técnicas Reunidas S.A. (Madrid, Spain).

\subsection{Supercritical water process}

Details for the continuous process used to depolymerize lignin in SCW were reported elsewhere. ${ }^{30}$ A simplified scheme of the process is shown in Fig. 8. Sudden Expansion Micro-Reactors (SEMR) consisting in $1 / 8^{\prime}$ inconel steel tubes with small internal diameters $(1.40-1.75 \mathrm{~mm})$ were used to achieve reaction times as short as 40 milliseconds. The lignin solution/suspension is continuously compressed and pumped up to the operation pressure, remaining at room temperature until the inlet of the reactor. When $\mathrm{NaOH}$ is required, it is premixed with the lignin stream. Heating is achieved by mixing the compressed room temperature lignin solution with a stream of hot pressurized water in the $\mathrm{T}$ junction. The product is depressurized through a high temperature needle valve (high-T valve), instantaneously cooling down the effluent down to ca. $180{ }^{\circ} \mathrm{C}$ and therefore stopping the reaction. To further reduce the temperature down to ambient, a cooler was set after the preheater used to preheat the SCW.

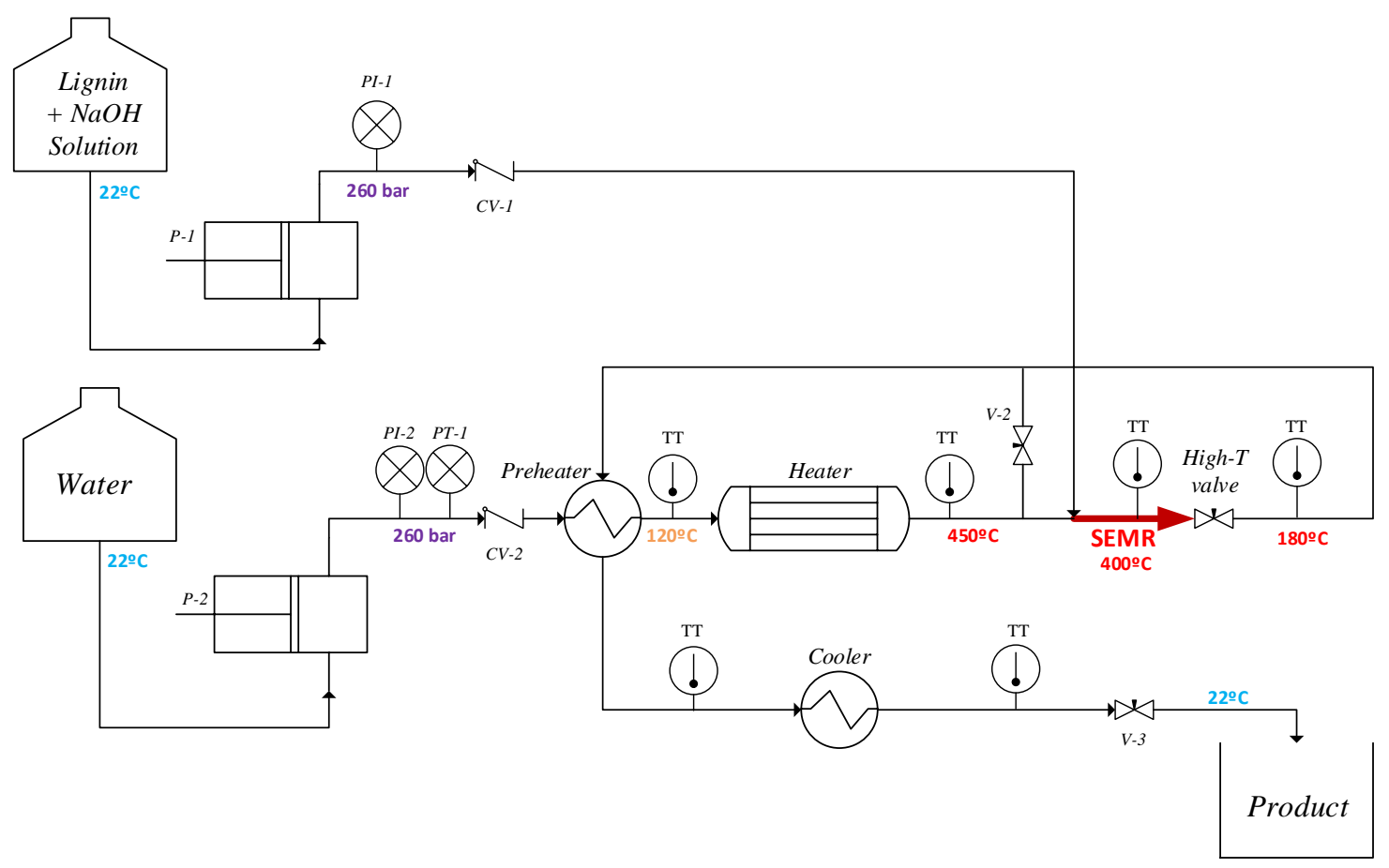

Figure 8. Scheme of the SCW plant. 
Lignin depolymerization was carried out at temperatures between $300^{\circ} \mathrm{C}$ to $400^{\circ} \mathrm{C}$ $\left(300,370,386\right.$ and $\left.400^{\circ} \mathrm{C} \pm 2.1^{\circ} \mathrm{C}\right)$ and $260 \pm 8.1$ bar, with reaction times between $60 \mathrm{~ms}$ and up to $4.6 \mathrm{~s}( \pm 0.01 \mathrm{~s})$ in the experiments at subcritical conditions. The reaction time was controlled by changing the total flowrate and the reactor volume. The lignin concentration at the inlet of the reactor was $1.0 \mathrm{~g} / 1$ for experiments without sodium hydroxide addition and $5.5 \mathrm{~g} / \mathrm{l}$ for experiments in alkaline medium $(2.5 \mathrm{~g} / \mathrm{l}$ and $15 \mathrm{~g} / \mathrm{l}$ in fed stream respectively). Kraft lignin is partially soluble in neutral water but that was not impediment as our pumps are able to pump suspensions. The concentration of $\mathrm{NaOH}$ at the inlet of the reactor was $0.2 \mathrm{M}$ for the experiments using base catalyst. The water flow was varied from $3.5 \mathrm{~L} / \mathrm{h}$ to $5.1 \mathrm{~L} / \mathrm{h}$ and the lignin flow was varied from 1.6 to $2.5 \mathrm{~L} / \mathrm{h}$. Different reactors were made with volumes between 0.4 and $12.3 \mathrm{~cm}^{3}$.

\subsection{Fractionation of depolymerization products}

Once the reaction finished, the reaction product was treated to recover the aromatic monomers of interest. A procedure was developed to separate the mixture into four fractions. ${ }^{2}$ A simplified scheme of this process is represented in Figure S1. Product mixture was acidified until $\mathrm{pH} 2$ in the case of experiments performed under alkaline medium ( $\mathrm{NaOH}$ addition). The water-insoluble solids were separated by centrifugation from the water-soluble compounds. Both fractions were extracted with ethyl acetate, obtaining four fractions labeled as light oil, heavy oil, char and aqueous residue after eliminating the solvent. Reproducibility of the experimental procedure was assessed by fractionating and analyzing three samples taken at the same conditions. The uncertainties obtained were: For light oil yield, 1.3\%; for heavy oil and char, 5\%; for monomer concentration, $<0.5 \%$.

The product of interest in this study was the light oil containing the phenolic compounds. The aqueous residue fraction contained the salts formed $\left(\mathrm{Na}_{2} \mathrm{SO}_{4}\right)$ during neutralization and it was not taken into account in this study.

\section{Conclusions}

Kraft lignin can be effectively depolymerized with hot and pressurized water at times below 500 ms using a Sudden Expansion Micro-Reactor. By controlling reaction time it is possible to avoid char formation due to repolymerization of the lignin molecule 
while maximizing the yield of bio-oil containing phenolics with high selectivity. At 300 ms, $386^{\circ} \mathrm{C}$ and adding $\mathrm{NaOH}$ as catalyst, the light oil yield was $60 \%$ with a concentration of key monomers close to $20 \%$. Analysis and quantification of the reaction products allowed distinguishing the different stages of the reaction: The depolymerization starts with a very rapid reactions that increases unsaturation degree of lignin and decreases its solubility in water. This step is followed by cleavage of $\mathrm{C}-\mathrm{O}$ and $\mathrm{C}-\mathrm{C}$ bonds up the optimum point. Monomers and oligomers starts to repolymerize at longer reaction times to yield char. Organic sulfur is incorporated to the latter at the last stages of the reaction.

The ultrafast SCW technology was used to directly treat a black liquor from a paper industry and it was found to be equally effective than when using commercial kraft lignin and even better that the kraft lignin isolated from the black liquor. A yield of $51 \%$ in light oil production with a selectivity up to $18 \%$ in key phenols were obtained.

These results represent a significant advance to the strategy of SCW depolymerization of lignin because black liquors can be directly treated without need of further treatment by a fast and intensified process while obtaining a high lignin conversion, relatively high monomer selectivity and low repolymerization. Moreover, the significant advantages that this novel process for lignin depolymerization presents (i.e. small reactors resulting in a feasible scale-up, cost and safety of the solvent and accurately time control to avoid undesirable condensation reactions) put this process on the top of the pyramid for the advancement and improvement of the future biorefinery.

\section{Acknowledgments}

The authors thank the Spanish Ministry of Economy and Competitiveness and Feder for funding the Project CTQ2016-79777-R and Técnicas Reunidas for supplying the Blackliquor and the Kraft-BL lignin. EP thanks Junta de Castilla y León for his postdoctoral fellowship. NAF thanks Spanish Education Ministry for her predoctoral fellowship FPU15/00409. Authors are grateful to the technicians for their assistance in the laboratory. 
${ }^{1}$ http://bio-based.eu/media/edd/2017/03/17-02-Bio-based-Building-Blocks-and-

Polymers-short version.pdf

${ }^{2}$ E. Pérez, C. O. Tuck. Quantitative analysis of products from lignin depolymerisation in high temperature water. Eur. Polym. J., 2018, 99, 38-48.

${ }^{3}$ R. Rinaldi, R. Jastrzebski, M. T. Clough, J. Ralph, M. Kennema, P. C. A. Bruijnincx, B. M. Weckhuysen. Paving the way for lignin valorisation: Recent Advances in Bioengineering, Biorefining and Catalysis. Angew. Chem. Int. Ed., 2016, 55, 8164-8215. ${ }^{4}$ A. Rahimi, A. Azarpira, H. Kim, J. Ralph, S. S. Stahl. Chemoselective Metal-Free Aerobic Alcohol Oxidation in Lignin. J. Am. Chem. Soc., 2013, 135, 6415-6418.

${ }^{5}$ L. Shuai, M. T. Amiri, Y. M. Questell-Santiago, F. Héroguel, Y. Li, H. Kim, R. Meilan, C. Chapple, J. Ralph, J. S. Luterbacher. Formaldehyde stabilization facilitates lignin monomer production during biomass depolymerization. Science, 2016, 354, 329-333.

${ }^{6}$ A. Rahimi, A. Ulbrich, J. J. Coon, S. S. Stahl. Formic-acid-induced depolymerization of oxidized lignin to aromatics. Nature, 2014, 515, 249-252.

${ }^{7}$ A. J. Ragauskas, G. T. Beckham, M. J. Biddy, R. Chandra, F. Chen, M. F. Davis, B. H. Davison, R. A. Dixon, P. Gilna, M. Keller, P. Langan, A. K. Naskar, J. N. Saddler, T. J. Tschaplinski, G. A. Tuskan, C. E. Wyman. Lignin Valorization: Improving Lignin Processing in the Biorefinery. Science, 344, 1246843

8 M. P. Pandey, C. S. Kim. Lignin Depolymerization and Conversion: A Review of Thermochemical Methods. Chem. Eng. Technol., 2011, 34, 29-41.

9 E. Pérez, C. O. Tuck, M. Poliakoff. Valorisation of lignin by depolymerisation and fractionation using supercritical fluids and conventional solvents. J. Supercrit. Fluids., 2018, 133, 690-695.

${ }^{10}$ Wahyudiono, M. Sasaki, M. Goto. Recovery of phenolic compounds through the decomposition of lignin in near and supercritical water. Chem. Eng. Process., 2008, 47, 1609-1619.

11 T. L.-K. Yong, Y. Matsumura. Reaction Kinetics of the Lignin Conversion in Supercritical Water. Ind. Eng. Chem. Res., 2012, 51, 11975-11988.

12 J. E. Miller, L. R. Evans, J. E. Mudd, K. A. Brown. Batch Microreactor Studies of Lignin Depolymerization by Bases. 2. Aqueous Solvents. In: SAND Report (SAND20021318). Sandia National Laboratories, 2002.

${ }^{13}$ V. M. Roberts, V. Stein, T. Reiner, A. Lemonidou, X. Li, J. A. Lercher. Towards Quantitative Catalytic Lignin Depolymerization. Chem. Eur. J., 2011, 17, 5939-5948. 
14 A. Oasmaa, A. Johansson. Catalytic hydrotreating of lignin with water-soluble molybdenum catalyst.

Energy Fuels, 1993, 7, 426-429.

${ }^{15}$ L. Shuai, B. Saha. Towards high-yield lignin monomer production. Green. Chem., 2017, 19, 3752-3758.

${ }^{16}$ M. J. Cocero, A. Cabeza, N. Abad, T. Adamovic, L. Vaquerizo, C. M. Martínez, M. V. Pazo-Cepeda. Understanding biomass fractionation in subcritical \& supercritical water. J. Supercrit. Fluids. 2018, 133, 550-565.

17 T. J. Mcdonough. The chemistry of organosolv delignification. TAPPI J., 1993, 76, 186-193.

${ }^{18}$ V. L. Pardini, R. R. Vargas, H. Viertler. Anodic Cleavage of Lignin Model Dimers in Methanol. Tetrahedron, 1992, 48, 1221-1228.

19 V. Roberts, S. Fendt, A. A. Lemonidou, X. Li, J. A. Lercher. Influence of alkali carbonates on benzyl phenyl ether cleavage pathways in superheated water. Appl. Catal. B-Environ., 2010, 95, $71-77$.

${ }^{20}$ X. Erdocia, R. Prado, M. A. Corcuera, J. Labidi. Base catalyzed depolymerization of lignin: Influence of organosolv lignin nature. Biomass Bioenergy, 2014, 66, 379-386.

${ }^{21}$ E. Jakab, O. Faix, F. Till, T. Székely. Thermogravimetry/mass spectroscopy study of six lignins within the scope of an international round robin test. J. Anal. Appl. Pyrolysis, 1995, 35, 167-179.

${ }^{22}$ W. Bühler, E. Dinjus, H. J. Ederer, A. Kruse, C. Mas. Ionic reactions and pyrolysis of glicerol as competing reaction pathways in near- and supercritical water. J. Supercrit. Fluids, 2002, 22, 37-53.

${ }^{23}$ D. A. Cantero, M. D. Bermejo, M. J. Cocero, Governing Chemistry of Cellulose Hydrolysis in Supercritical Water. ChemSusChem, 2015, 8, 1026-1033.

${ }^{24}$ T. Faravelli, A. Frassoldati, G. Migliavacca, E. Ranzi. Detailed kinetic modelling of the thermal degradation of lignins. Biomass Bioenergy, 2010, 34, 290-231.

25 G. González, J. Salvadó, D. Montané, Reactions of vanillic acid in sub- and supercritical water, J. Supercrit. Fluids., 2004, 31, 57-66.

${ }^{26}$ C. M. Martínez, T. Adamovic, D. A. Cantero, M. J. Cocero. Scaling up the production of sugars from agricultural biomass by ultrafast hydrolysis in supercritical water. $J$. Supercrit. Fluids, 2019, $242-250$. 
27 D. A. Cantero, L. Vaquerizo, F. Mato, M. D. Bermejo, M. J. Cocero. Energetic approach of biomass hydrolysis in supercritical water. Bioresource Technol., 2015, 179, 136-143.

${ }^{28}$ M. I. F. Mota, P. C. R. Pinto, J. M. Loureiro, A. E. Rodrigues. Recovery of Vanillin and Syringaldehyde from Lignin Oxidation: A Review of Separation and Purification Processes. Sep. Purif. Rev., 2016, 45, 227 - 259.

${ }^{29}$ A. Klemola, J. Tuovinen, (1989). Method for the production of vanillin U.S. Patent 4847422.

${ }^{30}$ D. A. Cantero, C. Martinez, M. D. Bermejo, M. J. Cocero. Simultaneous and selective recovery of cellulose and hemicellulose fractions from wheat bran by supercritical water hydrolysis. Green Chem., 2015, 17, 610 - 618. 